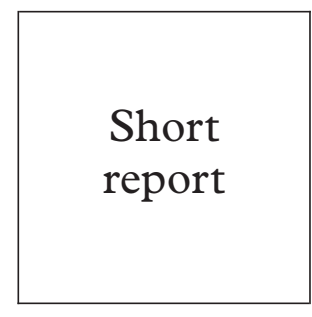

\title{
Post-exposure prophylaxis for human immunodeficiency virus: knowledge and experience of junior doctors
}

\author{
M Y Chen, E F Fox, C A Rogers
}

Objective: To assess the level of knowledge and experience of post-exposure prophylaxis (PEP) against human immunodeficiency virus (HIV) among junior doctors.

Methods: A questionnaire was sent to all junior doctors working in two major teaching hospitals in London.

Results: Most junior doctors had heard of PEP (93\%) but fewer were aware that it reduced the rate of HIV transmission ( $76 \%$ ). Only a minority of doctors $(8 \%)$ could name the drugs recommended in recent national guidelines and a significant proportion (43\%) could not name any. Almost one third (29\%) did not know within what period PEP should be administered. This was despite the fact that the majority of respondents (76\%) had experienced high risk exposure to potentially infective material at some stage in their careers and that a significant proportion (18\%) had sought advice about PEP following potential exposures.

Conclusions: This study demonstrates that the junior hospital doctors in our survey had inadequate knowledge of PEP against HIV despite being at risk of occupational exposure. (Sex Transm Inf 2001;77:444-445)

Keywords: HIV; post-exposure prophylaxis; needlestick injuries

\section{Introduction}

The rate of transmission of HIV following percutaneous exposure in the healthcare setting has been shown to be three per 1000 injuries. ${ }^{1}$ A case-control study performed by the Centres for Disease Control showed a $79 \%$ reduction in transmission with the administration of zidovudine. ${ }^{2}$ There are factors that limit the ability to conduct prospective placebo controlled trials assessing the efficacy of post-exposure prophylaxis (PEP) for HIV and, as a result, no such study has been performed. ${ }^{3}$

In the United Kingdom, guidelines for PEP were developed by the Expert Advisory Group on AIDS and issued in June 1997. As junior doctors are one group of healthcare professionals at risk of occupational exposure to HIV, we endeavoured to examine their levels of knowledge and experiences of PEP.

\section{Methods}

A postal survey was conducted in June 1998. Questionnaires were distributed to all house officers, senior house officers, and specialist registrars working at Guy's and St Thomas's Hospitals, London. There were 21 items listed on the survey. Knowledge of PEP was assessed by asking doctors if they had heard of PEP; if they were aware PEP reduced HIV transmission; which drugs were recommended and when these should be administered. Respondents were asked if they had encountered any percutaneous injuries or exposure of broken skin or mucous membranes to bodily fluids at any stage in their medical career. When asked if the patients involved were at high risk for HIV infection, doctors were asked to make their own assessment of that risk. Experience of PEP was determined by asking how often PEP had been sought; the reasons for not seeking PEP after potential HIV exposure; and doctors' experiences of PEP after antiretrovirals had been initiated. A second reminder questionnaire was sent to those who did not respond initially. A book prize was offered as an incentive to participate in the study.

\section{Results}

The response rate was $78 \%$ (273 completed questionnaires from a total of 350 junior doctors employed at both hospitals). Respondents had been practising for a mean of 6.6 years (range 0-22) and consisted of house officers, senior house officers, and specialist registrars working throughout various adult and paediatric, surgical, and medical specialties.

KNOWLEDGE OF POST-EXPOSURE PROPHYLAXIS Although 253 (93\%) doctors had heard of PEP for HIV, only 208 (76\%) were aware that PEP reduced the transmission rate of HIV. One hundred and eighteen (43\%) doctors could not name any of the drugs recommended for use after a high risk exposure. Twenty (7\%) knew that a three drug regimen was recommended, although only 23 (8\%) could name the specific drugs. Although 89 (33\%) were aware PEP should be commenced within 1 hour of exposure, 80 (29\%) respondents did not know how quickly PEP should be given.

EXPERIENCE OF POST-EXPOSURE PROPHYLAXIS Two hundred and eight $(76 \%)$ of those surveyed reported exposure to potentially infectious material on one or more occasions. These included 200 percutaneous injuries, 48 episodes of broken skin exposure to bodily
Accepted for publication 26 June 2001 
Table 1 Reasons given for not seeking advice about PEP in those who reported potential HIV exposure*

\begin{tabular}{lll}
\hline Reason & $\begin{array}{l}\text { Number of } \\
\text { respondents }\end{array}$ & $\%$ \\
\hline The patient was at low risk for HIV & 125 & 79 \\
Did not have time or too busy & 26 & 16 \\
Did not think or know about it & 25 & 16 \\
Did not know how or where to get it & 8 & 5 \\
Worried about side effects of drugs & 6 & 4 \\
Did not think it would work & 5 & 3 \\
Exposure predated guidelines on PEP & 14 & 9 \\
\hline
\end{tabular}

^159 respondents gave 209 reasons.

fluids, and 76 episodes of mucous membrane exposure. Twenty four doctors $(9 \%)$ reported a total of 32 exposures to a patient who they considered to be at high risk of HIV infection.

Forty nine $(18 \%)$ respondents had sought advice about PEP within the hospital at some stage in their career. The most common source from which advice was sought was the occupational health department $(53 \%)$. The reasons given by those who had not sought advice are shown in table 1 . Of the 49 doctors who sought advice, 12 were offered PEP. Six commenced PEP but only two completed the course. The four who discontinued their antiretroviral therapy did so because the patient was later thought to be at very low risk or had ultimately tested HIV negative.

\section{Discussion}

The UK Department of Health has issued guidelines which recommend that PEP should be considered when there has been exposure to material known, or strongly suspected to be, infected with HIV. ${ }^{5}$ The combination suggested is zidovudine, lamivudine, and indinavir. This should be taken as soon as possible, and ideally within 1 hour, for a 4 week period. The US Public Health Service recommends a 4 week course of zidovudine and lamivudine, with the addition of indinavir or nelfinavir for higher risk exposures. ${ }^{5}$ All healthcare workers should be aware of the risks from occupational exposure so that the delay in seeking advice is minimised.

This study demonstrates that doctors in training in our trust had an inadequate level of knowledge about PEP. Some were not aware that PEP for HIV existed. Of those who had heard of PEP, only a minority could name the drugs recommended. A substantial proportion of doctors were uncertain about how quickly the drugs should be administered.

This poor level of knowledge was apparent despite the fact that the majority of respondents reported exposures to potentially infective material at some stage of their careers.
A proportion of exposures will have predated the 1997 UK guidelines; however, only $9 \%$ of respondents cited this as a reason for not obtaining PEP. Another survey conducted among junior doctors in London highlighted the underreporting of needlestick injuries in this group and the low level of glove usage in the performance of venepuncture, intravenous cannula insertion, and arterial blood gas sampling. ${ }^{6}$ Up until December 1997, there had been 95 definite and 191 possible cases of occupationally acquired HIV infection among healthcare workers. Most of these were from developed countries, with no reports at all from most developing countries. ${ }^{7}$ Forty six $(16 \%)$ of these occurred in doctors or medical students. The combination of high risk of exposure coupled with poor knowledge of PEP is of serious concern.

We feel that it is of great importance to improve the level of knowledge about occupational exposure to HIV among junior hospital doctors. This should include education on how to prevent exposure, as well as information on first aid, who to contact, and the urgency with which advice on PEP needs to be sought. This should start at the undergraduate level, as medical students in the clinical setting will also be at risk. Education should be included in all induction programmes for junior doctors at the start of new posts. Each hospital trust should have a written policy on PEP, which is easily accessible to doctors, especially after hours. Such measures could save unnecessary anguish.

We thank all the doctors at Guy's and St Thomas's Hospitals who participated in our survey.

Contributors: MYC and EFF were responsible for data collection and writing of the manuscript; CAR provided guidance on the development of the study. A $£ 150$ book voucher sponsored by GlaxoWellcome was offered as an incentive to doctors for returning questionnaires.

The authors declare no conflict of interest.

1 Gerberding JL. Management of occupational exposure to bloodborne viruses. N Engl F Med 1995;332:444.

2 Centres for Disease Control. Case-control study of HIV seroconversion in health-care workers after percutaneous exposures to HIV-infected blood-France, United Kingdom and United States, January 1988-August 1994. MMWR 1995;44:929.

3 Henderson D. Post-exposure chemoprophylaxis for occupational exposures to human immunodeficiency virus. $\mathscr{F} A M A$ 1999;281:931-6.

4 Expert Advisory Group on AIDS. Post-exposure prophylaxis for health care workers exposed occupationally to HIV. London: Department of Health, 1997.

5 Centres for Disease Control and Prevention. Public Health Service guidelines for the management of health-care worker exposures to HIV and recommendations for

post-exposure prophylaxis. $M M W R$ 1998;47(RR-7):1-33. reporting of needlestick injuries by junior hospital medical reporting of needlestick injuries by junior hosp
staff. Ann R Coll Surg Engl 1998;80:439-41.

7 Occupational transmission of HIV infection. Commun Dis Rep Wkly 1998;8:193. 